\title{
Reusable plasmonic substrates fabricated by interference lithography: a platform for systematic sensing studies
}

\author{
Thomas Siegfried, ${ }^{a}$ Martin Kind, ${ }^{b}$ Andreas Terfort, ${ }^{\text {b }}$ Olivier J. F. Martin, ${ }^{c}$ \\ Michael Zharnikov, ${ }^{d}$ Nirmalya Ballav ${ }^{e_{*}}$ and Hans Sigg ${ }^{a_{*}}$
}

Surface-enhanced Raman scattering (SERS) has become increasingly popular in the scientific and industrial communities because of its analytical capabilities and potential to study fundamentals in plasmonics. Although under certain conditions extremely high sensitivity is possible, the practical use of SERS is frequently limited by instability and poor reproducibility of the enhancement factor. For analytical applications or for comparative measurements to enable the distinction between electromagnetic and chemical enhancement, the development of standardized and recyclable SERS substrates, having uniform and persistent performance, is proposed. To this end, we have fabricated periodic nanoslit arrays using extreme ultraviolet lithography that provide average large $\left(2 * 10^{6}\right)$ and homogeneous SERS enhancement factors with a spot-to-spot variability of less than $3 \%$. In addition, they are reusable without any degradation or loss of enhancement. The fabrication of such arrays consists of two steps only, lithographic patterning followed by metal evaporation. Both processes may be performed over areas of several square mm on any planar substrate. The sensor capabilities were demonstrated by substrates with monomolecular films of several different thiols. The concept of reusable SERS substrates may open a powerful platform within an analytical tool and in particular for systematic SERS studies for the investigation of fundamental parameters such as chemical enhancement, surface selection rules, and molecular alignment. Copyright $\odot 2012$ John Wiley \& Sons, Ltd.

Supporting information may be found in the online version of this article.

Keywords: surface-enhanced Raman scattering; reusability; reproducibility, nanofabrication; analytical tool

\section{Introduction}

Thanks to its strong signal enhancement in the vicinity of metal nanostructures, surface-enhanced Raman scattering (SERS) is emerging as one of the most prominent plasmonic sensing techniques. ${ }^{[1-3]}$ Because of the increased sensitivity in the detection of characteristic vibrational modes associated with a specific (surface-adsorbed) analyte, SERS has established itself as a labelfree detection technique that may be applied to almost any adsorbate down to concentrations of a single molecule. A large variety of SERS-active substrates, such as roughened or patterned metallic surfaces and nanoparticle-based systems have been fabricated. ${ }^{[3-6]}$ Among these substrates, Au-based and Ag-based nanostructures with sharp edges (so called 'hot-spots') are preferred because of their biocompatibility ${ }^{[7]}$ and their extremely high electromagnetic fields arising from the excitation of local surface plasmon resonances. However, such 'hot-spots' associated with rough or nanoparticle-based substrates are randomly distributed at the nano scale such that these substrates frequently respond in an irreproducible way. ${ }^{[8]}$ This lack of control renders such substrates rather useless for systematic studies and the characterization of molecular assemblies. ${ }^{[9]}$

In fact, an ideal analytical platform for cost-effective and reproducible measurements would ideally consist of a reusable and standardized substrate. Subsequently, one could identify and separate fundamental parameters such as the chemical enhancement from a systematic study of different adsorbed species and physical surroundings using the SAME substrate. Of course, this approach requires an effective cleaning procedure and robust substrates, so that the substrate sensitivity is not altered during repeated cycles of use. ${ }^{[10]}$ Substrate reusability has previously been reported with the use of random particle-based or self-ordered array substrates ${ }^{[11]}$ and protective oxide layers ${ }^{[12]}$ that can prevent metal contamination and also strongly reduce the enhancement factor. Custom tunability of the plasmon resonance is however limited in these substrates and also the spot to spot deviation is found to be well above $12 \%{ }^{[11,13]}$

\footnotetext{
* Correspondence to: Hans Sigg, Laboratory for Micro- and Nanotechnology, Paul Scherrer Institut, 5232 Villigen, Switzerland. E-mail: hans.sigg@psi.ch

* Nirmalya Ballav, Department of Chemistry, Indian Institute of Science Education and Research, 411021 Pune, India.

E-mail: nballav@iiserpune.ac.in

a Laboratory for Micro- and Nanotechnology Paul Scherrer Institut, 5232, Villigen, Switzerland

b Institut für Anorganische und Analytische Chemie, Goethe-Universität Frankfurt 60438, Frankfurt, Germany

C Nanophotonics and Metrology Laboratory EPFL, 1015 Lausanne, Switzerland

d Angewandte Physikalische Chemie, Universität Heidelberg, 69120 Heidelberg, Germany

e Department of Chemistry, Indian Institute of Science Education and Research, 411021 Pune, India
} 
A suitable way to meet the demand for high signal strength and reproducibility is lithography combined with sophisticated pattern design. ${ }^{[14,15]}$ Electron-beam lithography is especially attractive because of its flexibility and ultimate lateral resolution allowing fabrication of complex nanopatterns. ${ }^{[16]}$ However, the serial writing process of e-beam lithography makes this method economically unattractive. The combination of the precision of e-beam lithography with parallel, large-scale fabrication methods of nanopatterns employing extreme ultraviolet (EUV) interference lithography is proposed as the winning approach. It offers high-resolution patterning with a half-pitch down to about $10 \mathrm{~nm} \cdot{ }^{[17]}$ We fabricated nanoslit-based Au substrates exhibiting a high and reproducible SERS signal enhancement over several $\mathrm{mm}^{2}$ while withstanding multiple cleaning cycles. To quantify the reusability of this new substrate, we chose thiol-anchored chemisorbed monolayers as the analyte. We observed a vanishing dependence of the thiol adsorption probability on the metal substrate upon repeated cleaning cycles. The constant successive SERS signals prove that the cleaning is neither altering nor modifying the geometry of the nanoslits. No noticeable changes in the enhancement factor were observed after several successive cleaning/self-assembly cycles.

\section{Experimental}

\section{Materials and preparation}

All solvents and chemicals were purchased from Sigma-Aldrich $\mathrm{GmbH}$ and used without further purification. The studied selfassembled monolayer (SAM) precursor molecules biphenylthiol (BP0), 4,4'-terphenyl-4-thiol (TP0), 4,4'-terphenyl methanethiol (TP1), and 4,4'-perfluoroterphenyl methanethiol (FTP1) were custom-synthesized following the published protocols. ${ }^{[18,19]}$ SAM layers were formed by immersion of freshly patterned or cleaned plasmonic sensor substrates into a $1 \mathrm{mM}$ solution of the respective precursor in tetrahydrofuran (THF) for $>1 \mathrm{~h}$ at room temperature. After immersion, the samples were carefully rinsed with pure THF, distilled water and ethanol, and blown dry in a nitrogen stream.

\section{Nanofabrication of nanoslit substrates}

The nanocavity substrates were fabricated using shadow evaporation of gold ( $99.99 \%$ purity, purchased from Balzers) on the prepatterned layer of photoresist (hydrogen silsesquioxane, HSQ) on float glass chips. The 80-nm-thick HSQ film was exposed using EUV interference lithography ${ }^{[20]}$ at the Swiss Light Source to form a line pattern with period $250 \mathrm{~nm}$ over one $\mathrm{mm}^{2}$. Although this technique is rather unique, it has its main advantages in the exposure time (roughly $4 \mathrm{~s}$ ) for the high resolution pattern of large areas $\left(\mathrm{mm}^{2}\right)$. The HSQ layer was developed for $60 \mathrm{~s}$ in a $25 \%$ tetramethylammonium hydroxide solution and used without further liftoff treatments. Gold and chromium ( $1 \mathrm{~nm}$ acting as adhesion layer) were thermally evaporated at an angle of $60^{\circ}$ from the surface normal and with an azimuthal orientation perpendicular to the nanoslits. The base pressure was $4^{*} 10^{-6} \mathrm{mbar}$. Further details on the fabrication process can be taken from the literature. ${ }^{[21]}$

\section{Instrumentation}

Reflection spectra were recorded on a Sentech FTP advanced spectrometer attached to a Zeiss optical microscope with a 20 -fold objective. The spectra were normalized with a reference spectrum on the same substrate at a continuous gold layer without the nanoslit pattern to account for metal thickness and roughness effects. Because nanoslit arrays are highly polarization dependent, the incident light was polarized, that the electric field was perpendicular to the nanogap expansion. For opposite polarization the pattern exhibits no resonance and subsequently no SERS enhancement. ${ }^{[21]}$ Raman spectra were taken on a Horiba LabRam HR with a grating resolution of 600 lines $\mathrm{mm}^{-1}$. The excitation source was a HeNe laser operating at $633 \mathrm{~nm}$ and the incident laser power was fixed at $2 \mathrm{~mW}$. The system was equipped with a constantly rotating laser spot that reflected the beam within an area of $10 \times 10 \mu \mathrm{m}^{2}$, further minimizing substrate degradation and photobleaching of the analyte to a maximal level of $10 \%$ during $100 \mathrm{~s}$ of illumination. The laser was focused onto the dried substrate with an Olympus $\times 50$ long distance objective (numerical aperture, NA 0.5). An integration time of only $1 \mathrm{~s}$ was sufficient to obtain high quality Raman spectra and 16 spectra were taken over a patterned area of $600 \times 300 \mu \mathrm{m}^{2}$ for statistical evaluation. Baseline corrections were carried out to correct the optical background signal from the substrate. The SERS intensity of all subsequent figures corresponds to the highest Raman peak intensity around $1600 \mathrm{~cm}^{-1}$ of the analytes and is normalized for comparison of different species.

\section{Substrate cleaning}

The substrates were cleaned using an ultraviolet ozone (UVO) cleaner 42 from Jelight Company, Inc. The typical illumination time was $20 \mathrm{~min}$ for BP0, TP0, and FTP1, and $30 \mathrm{~min}$ for TP1. The distance between the substrate and lamp was $15 \mathrm{~mm}$ with an irradiation power of $0.28 \mathrm{~W} \mathrm{~cm}^{-2}$. After illumination, the substrates were thoroughly washed in THF, ethanol, and distilled water to remove the remaining contaminants and blown dry in a nitrogen stream.

\section{Raman spectra simulation}

Density functional theory calculations of the Raman spectra: the calculations were performed at the Center for Scientific Computing (CSC) of the Goethe University Frankfurt using the GaUSSIAN 09 program package. ${ }^{[22]}$ The optimal functional (Becke, threeparameter, Lee-Yang-Parr) and basis set (6-311G*) was selected by comparing the conventional Raman spectra of BPO (recorded with a Renishaw RM-1000 micro-Raman spectrometer at the department of geosciences/geography of the Goethe University of Frankfurt) with the calculated spectra. This combination was used thereafter for the calculation of the Raman spectra of all other thiol molecules investigated in this study. The calculated spectra were used to aid the band assignment of the SERS peaks. The wavenumbers in all calculated spectra were scaled by a factor of 0.975 to further account for overestimated energies of the respective vibrational modes.

\section{Results and discussion}

Gold was used as the active plasmonic material, because this noble metal does not form protective surface oxide layers and can be cleaned reproducibly (see below). The geometry of the pattern (Fig. 1) was designed in such a way that the energy of the plasmon resonance was in between the excitation line and the detected Raman signal, as shown in Fig. 1(c). Such configuration is known to provide the highest SERS enhancements. ${ }^{[23]}$ In a previous study 


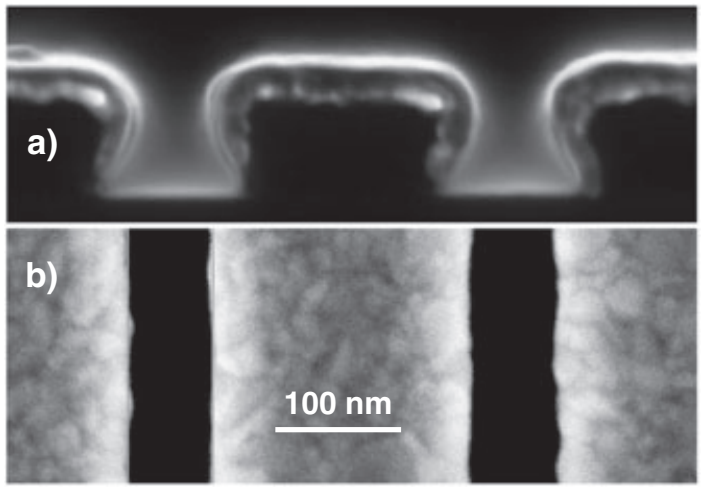

c)

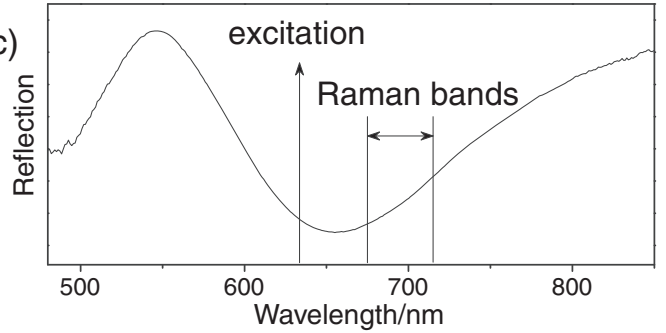

Figure 1. (a) Cross-section and (b) top-view scanning electron microscopy images of the EUV fabricated nanoslit pattern. The pattern is written in HSQ photoresist ( $80 \mathrm{~nm}$ thickness) and has a periodicity of $250 \mathrm{~nm}$. The lines are covered by Au ( $35 \mathrm{~nm}$ thickness) serving as active metal. The gap size between the Au lines is $60 \mathrm{~nm}$. (c) Normalized optical reflection spectrum of a typical nanoslit pattern with an incident polarization of the electric field perpendicular to the gap expansion. The Raman excitation wavelength is $633 \mathrm{~nm}$ while the detected bands are wavelength shifted by $1100-1700 \mathrm{~cm}^{-1}$.

it was shown that the nanogap dimension strongly alters the electric near-field intensity of the pattern and the strength of the SERS enhancement. ${ }^{[21]}$

As a representative test system for SERS experiments, we have selected a series of arene-based monomolecular films with a thiol headgroup as a suitable anchor for the Au substrate. The thus obtained self-assembled monolayers (SAMs) form a well-defined and homogeneous molecular adsorbate, providing a good platform for monitoring the SERS performance of our nanoslit substrate. In addition, SAMs are frequently used as building blocks of sensors and nanoscale assemblies, and are of great interest for lithography, nanofabrication, and organic electronics on their own. ${ }^{[24,25]}$

The SAM precursors under study are biphenylthiol (BP0), 4,4'-terphenyl-4-thiol (TP0), 4,4'-terphenyl methanethiol (TP1), and 4, $4^{\prime}$-perfluoroterphenyl methanethiol (FTP1) (see Fig. 2). All these molecules have been previously shown to form well-ordered, densely packed and contamination-free SAMs on thermally evaporated Au thin film substrates - as in the present study. ${ }^{[18,26,27]}$

Surface-enhanced Raman scattering spectra of the target SAMs on the nanoslit substrate are presented in Fig. 3(a). The spectra were recorded successively (system after system) on the same substrate area, with the following substrate cleaning step in between each measurement: the SAM-covered substrate was exposed to ultraviolet light, which generated chemically active ozone species. It is well-known that the SAM constituents are decomposed upon such a treatment while the thiolate headgroups ( $\mathrm{S}-\mathrm{Au}$ ) become sulphonates $\left(\mathrm{SO}_{3}-\mathrm{Au}\right)$, which are weakly bond to the substrate. The resulting physisorbed species can then be easily removed with a solvent rinsing procedure or exchanged for another SAM precursor upon immersion into the solution

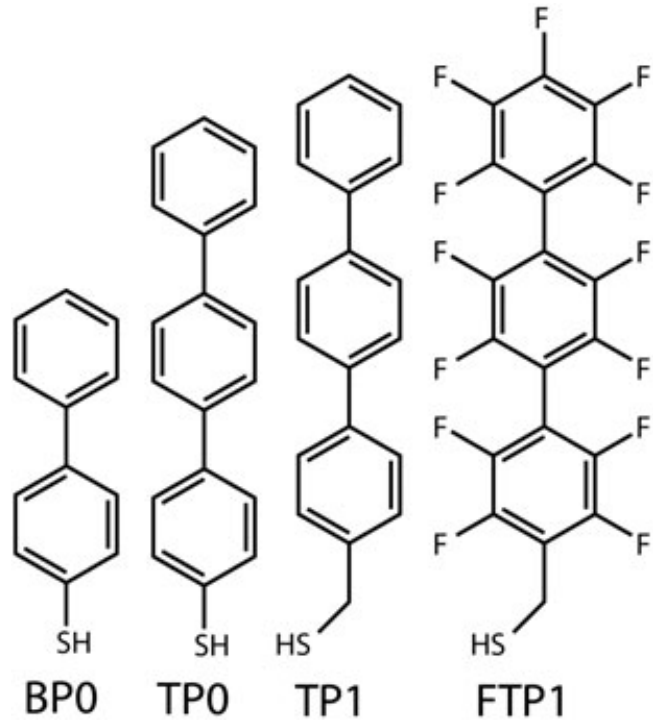

Figure 2. Structure of the SAM precursors used in this study, along with the respective abbreviations.

a)

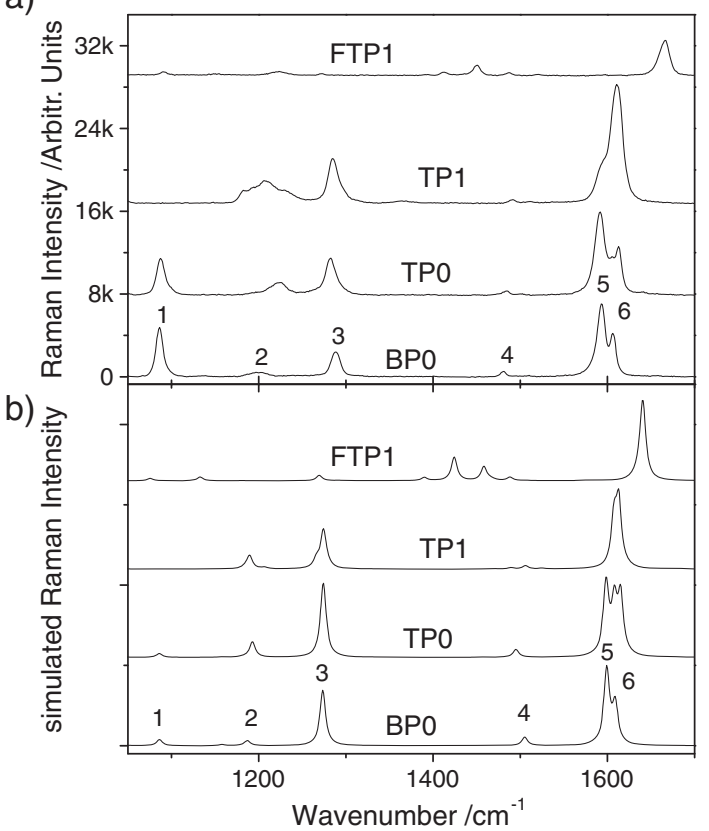

Figure 3. Comparison of (a) typical experimental SERS spectra of the monomolecular films with (b) the simulated spectra of the isolated SAM precursors. A baseline correction was applied to the experimental spectra and normalized intensities were applied to the simulated spectra.

containing these moieties. ${ }^{[28]}$ This guarantees the reusability of the substrate, shown in Fig. 5 , if the gold morphology is not altered during ultraviolet irradiation.

The SERS spectra in Fig. 3(a) possess a very high signal-to-noise ratio, exhibiting intense characteristic vibration modes of the target molecular assemblies. In the case of BP0, TP0, and TP1, the spectra are dominated by the ring breathing modes at $\sim 1280 \mathrm{~cm}^{-1}$ and $1600 \mathrm{~cm}^{-1}$, with the latter mode consisting of three components related to the individual rings. These peaks are accompanied by weaker features at $1200 \mathrm{~cm}^{-1}$ and $1500 \mathrm{~cm}^{-1}$ associated with the bending modes of the $\mathrm{CH}$ bond. In addition, in the spectra of BPO 
and TPO, there is a relatively strong peak at $\sim 1080 \mathrm{~cm}^{-1}$ that can be assigned to the stretching mode $\left(v_{\mathrm{CS}}\right.$ of the $\mathrm{S}-\mathrm{C}$ (ring) bond. ${ }^{[29]}$ Expectedly, this mode only exists if there is no substituent between the $S$ atom and the phenyl ring - but not for TP1/Au and FTP1/Au.

The difference in the distribution of the spectral weight within the $v_{\mathrm{CC}}$ mode $\left(1600 \mathrm{~cm}^{-1)}\right.$ for the BPO/TPO and TP1 cases is presumably related to the enhancement of the contribution of the bottom ring (see Fig. 3) for BPO and TPO because this moiety is in direct neighborship to the $\mathrm{S}$ atom, which has a higher polarizability than a $\mathrm{C}$ atom $\left(-\mathrm{CH}_{2}\right.$ group of TP1). For FTP1, the appearance of the corresponding ring breathing modes at higher wavenumbers (around $1450 \mathrm{~cm}^{-1}$ and $1665 \mathrm{~cm}^{-1}$ ) indicates a clear signature of the aromatic ring substitution by strong electron withdrawing fluorine atoms. ${ }^{[26]}$

The vibrational modes of the experimental SERS spectra were confirmed by theoretical simulations of the isolated SAM precursors. The obtained simulated spectra are presented in Fig. 3(b). Excellent agreement of most peak positions and relative intensities between the experimental and simulated spectra was found for all the studied systems, which is additionally illustrated in Table 1 by a detailed comparison of the peak positions and intensities for the BPO case (and additionally in Fig. S1 and Table S1 for all other precursors). Surface selection rules can play an important role in weakening of Raman bands because of an unfavorable local field polarization and molecular alignment. ${ }^{[8,30,31]}$

Comparison of surface-enhanced Raman spectra with conventional Raman spectra of the investigated substances has showed no noteworthy changes in the relative intensities of the vibrational bands. Interestingly, there is a strong additional amplification (by a factor of $\sim 20$ ) of the $v_{C S}$ stretching mode for the SERS system compared with the theory of neat Raman, occurring also for TPO. This amplification stems presumably from a change in the molecule polarizability and from particular high influence of the chemical enhancement in the $v_{C S}$ case. The latter enhancement could be associated with the charge transfer upon the formation of the substrate-S anchor and with the respective change in the electronic configuration of the $\mathrm{C}$ (ring) $-\mathrm{S}$ bond. ${ }^{[32]}$ Note that the $\mathrm{S}-\mathrm{H}$ bending mode exhibited in the simulated spectra at about $920 \mathrm{~cm}^{-1}$ (not shown) was not observed in the experiments, thereby proving the expected abstraction of $\mathrm{H}$ and covalent attachment of the SAM precursors to the substrate via the thiolate $(\mathrm{S}-\mathrm{Au})$ bond.

Figure 4 compares the Raman spectra of patterned and unpatterned areas, and a normal Raman spectrum of the precursor TP1 solution in THF. While the normal Raman spectrum is strongly dominated by the solvent peaks $\left(914,1030,1240\right.$, and $\left.1480 \mathrm{~cm}^{-1}\right)$,

Table 1. Wavenumber positions (WN), relative intensities, and assignments of the Raman peaks for the BP0 case. Experimentally determined values (exp.) are compared with the simulation results (sim.)

\begin{tabular}{cccccc} 
Nr. & $\begin{array}{c}\text { exp. } \\
\text { WN/cm }\end{array}$ & $\begin{array}{c}\text { exp. } \\
\text { Intensity }\end{array}$ & $\begin{array}{c}\text { sim. } \\
\text { WN/cm }\end{array}$ & $\begin{array}{c}\text { sim. } \\
\text { Intensity }\end{array}$ & Assignment \\
\hline 1 & 1084 & $\mathrm{~s}$ & 1086 & 7 & $v_{\mathrm{CS},} \beta_{\mathrm{CH}}{ }^{a}$ \\
2 & 1198 & W, broad & 1187 & 6 & $\beta_{\mathrm{CH}}$ \\
3 & 1286 & $\mathrm{~m}$ & 1273 & 69 & $v_{\mathrm{CC},} \beta_{\mathrm{CH}}$ \\
4 & 1480 & $\mathrm{w}$ & 1480 & 11 & $\beta_{\mathrm{CH}}$ \\
5 & 1593 & $\mathrm{~s}$ & 1599 & 100 & $v_{\mathrm{CC},} \beta_{\mathrm{CH}}$ \\
6 & 1605 & $\mathrm{~m}$ & 1609 & 52 & $v_{\mathrm{CC},} \beta_{\mathrm{CH}}$ \\
\hline
\end{tabular}

${ }^{a} v$ are stretching modes and $\beta$ are in-plane bending modes. Signal strength: s, strong; $\mathrm{m}$, medium; $\mathrm{w}$, weak.

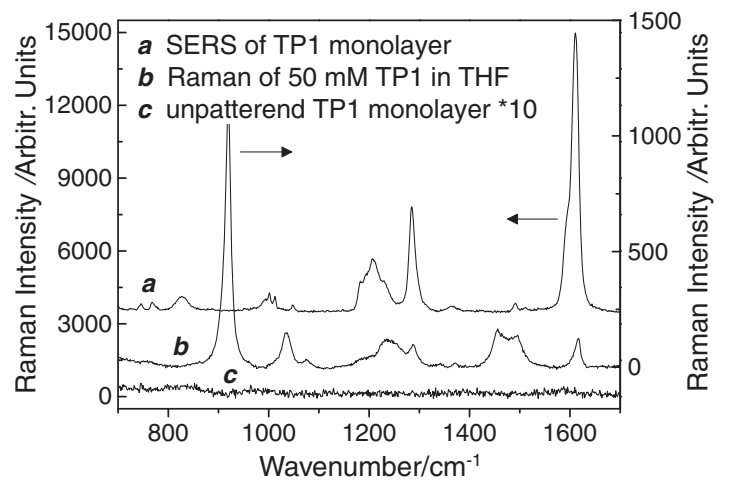

Figure 4. SERS spectra for a TP1 monolayer (a) at a patterned and (c) unpatterned Au substrate. (b) Normal Raman spectrum of a 50-mM TP1 solution in THF. The normal Raman was recorded with an $\times 10$ (NA 0.2) objective at a tenfold increased intensity $(20 \mathrm{~mW})$. A baseline correction was applied to all spectra.

the $v_{\mathrm{CC}}$ mode of TP1 at $1609 \mathrm{~cm}^{-1}$ is clearly separated. Significantly, no SERS peaks could be detected from unpatterned areas of the same substrate (separated $10 \mu \mathrm{m}$ from the pattern) covered by the SAMs, even with tenfold higher excitation intensity. This observation leads to the conclusion that the SERS 'hot-spots' are located solely at the nanoslit arrays and surface roughness does not contribute to their generation. In a previous publication we have shown the polarization dependency of the SERS signal on nanogap arrays. ${ }^{[21]}$ Full signal was obtained when the electric field is perpendicular to the nanogap expansion (TE), while no SERS signal could be found for the TM mode. Despite ambiguities for the determination of SERS enhancement factors, we have calculated an average enhancement factor following a conservative routine described in the literature. ${ }^{[33]}$ The enhancement factor was calculated on the basis of the $1609 \mathrm{~cm}^{-1}$ peak of TP1, with SERS spectra recorded with an $\times 50$ (NA 0.5) objective and normal Raman spectra recorded with an $\times 10$ (NA 0.25) objective from a $50 \mathrm{mM}$ solution at tenfold increased excitation intensity. The monolayer packing density was expected to be similar to benzenethiol, $6.8^{*} 10^{14} \mathrm{~cm}^{-2},{ }^{[34]}$ and leads to a precursor density of $3.2 * 10^{6}$ for the SERS measurement, considering a laser spot size of $0.8 \mu \mathrm{m}$. Considering a focal volume of $10.6 \mathrm{pL}$, the molecule density of the normal Raman measurement was calculated to be $3.2^{*} 10^{8}$. Together with the area integrated Raman peaks of 190 for the normal Raman spectrum and 290000 for the SERS spectra, an area average enhancement factor of $2^{*} 10^{6}$ was obtained.

\section{Cleaning and reusing SERS substrates}

The dynamics of the self-assembly and cleaning steps was monitored to understand and optimize the substrate performance. During the self-assembly step, a logarithmic growth of the SERS intensity with immersion time was found, as illustrated in Fig. 5(a) by the example of TPO (left panel). Assuming that the SERS intensity scales predominately linear with the number density of chemisorbed molecules (only valid for up to monolayer coverage and while EM and chemical enhancement are constant), we conclude that the formation of the monomolecular film was completed after $4 \mathrm{~min}$. Longer immersion (up to $24 \mathrm{~h}$ ) did not result in a change of the SERS intensity and respectively the molecular coverage (a structural rearrangement or alignment was however 
a)

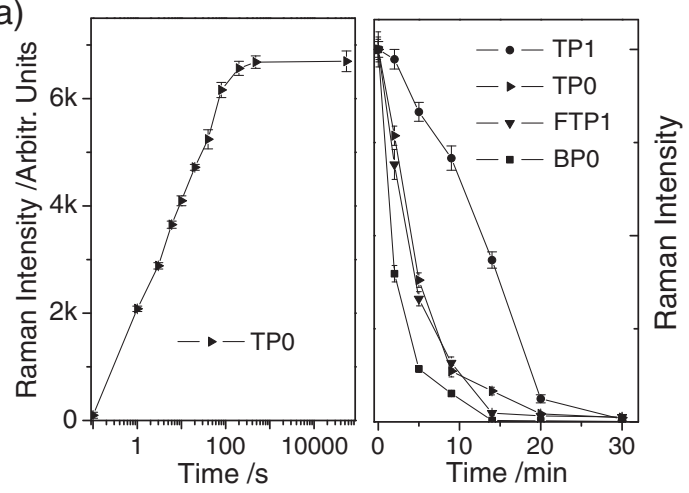

b)

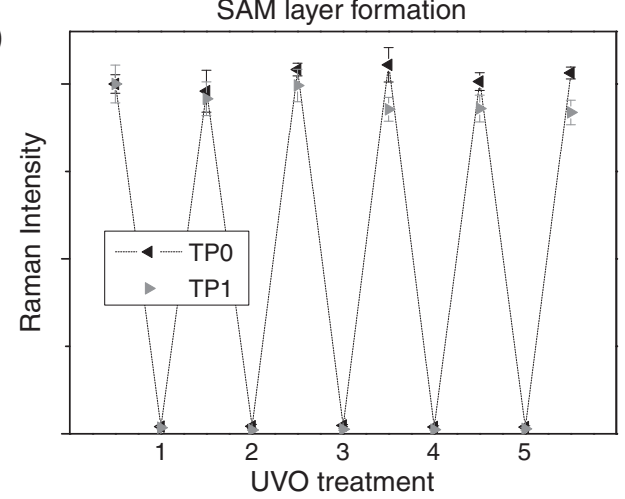

Figure 5. (a) SERS intensity of the strongest peak of the $v_{\mathrm{Cc}}$ mode around $\sim 1600 \mathrm{~cm}^{-1}$ during the formation of the TPO SAM on the nanoslit substrate (left panel) and for all precursors during UVO cleaning of the SAM covered substrate (right panel). The data points were recorded ex situ. (b) SERS intensity of the $v_{\mathrm{Cc}}$ mode around $\sim 1600 \mathrm{~cm}^{-1}$ for the molecules TPO and TP1 during repeated cycles of substrate cleaning and monolayer reformation using the exact same pattern. Error bars correspond to the standard deviation of 16 spatially separated Raman measurements.

possible). Note that the intensity of the observed peaks, i.e. the SERS enhancement, was highly reproducible over the entire patterned substrate area; we have found an excellent standard deviation of below 3\% throughout all measurements from the recorded spectra of 16 spatially separated regions on the sensor.

The recorded dynamics of the substrate cleaning process exhibited an exponential decay of the SERS intensity, as seen in Fig. 5(a) (right panel) with full spectra given in Figs S2 and S3. After 20 min of treatment, the detectable SERS intensities were well below $3 \%$ of the original value. Among the used molecules, only TP1 differed in the cleaning dynamics, with a longer time necessary for the cleaning, which could be caused by the especially tight molecular packing for this particular system ${ }^{[27]}$ hindering the penetration of the reactive ozone species to the thiolate-substrate interface. The SERS spectra of cleaned substrates (Fig. 5(b)) prove that all SAM constituents and their fragments could be removed from the substrate, which makes the sensor reusable for SERS measurements on further molecular films of different analytes, in case the pattern morphology is not influenced by the cleaning process.

In the following, we demonstrate the reusability of the nanoslit substrate, shown in Fig. 5(b). By alternating the self-assembly and the UVO cleaning steps on exactly the same substrate, we observed that it could withstand at least six cycles without any noticeable change in the obtained SERS spectra. The SERS peaks around $1600 \mathrm{~cm}^{-1}$ were compared for the SAM layers of TPO and TP1 during repeated cleaning and assembly cycles, shown in Fig. 5(b) with full spectra given in Figs S4 and S5. In particular, the substrate used for the TP0 showed an excellent persistence of the average SERS enhancement with a deviation after each cleaning/selfassembly step below $3 \%$. Building on these results, one could expect a reusable substrate for many more than the analyzed six cycles. This excellent reusability also indicates that the metal surface is not altered and contaminated during repeated cleaning steps so that the adsorbed molecules can still be well ordered and assemble with a similar packing density as for the pristine gold substrate. We think that thiols are one of the difficult cases in the sense of SERS substrate reusability because of the strong bonding and the easiness to cleave the $\mathrm{S}-\mathrm{C}$ bond in the course of the cleaning procedure (the substrate will then be passivated with atomic sulphur). We believe that the reusability of the presented SERS-active substrates will be equally high in the case of weakly bound adsorbates, but cannot exclude that specific problems can arise for some of these systems.

Similar signal stability was also obtained for TP1, although there was a slight decrease observed after the third cycle. This decrease could be related to heating of the substrate during the UVO exposure, leading to microscopic changes in the metal morphology. Scanning electron microscopy images taken before and after the cleaning steps could however not reveal changes in the nanogap dimension. Note that to ensure a complete detachment of TP1, the UVO exposure time had to be 10 min longer than that for TPO, producing additional heating of the substrate. This can probably be compensated by external cooling, which however was not investigated in the present study. We do not claim that the demonstrated reusability is the exclusive property of our pattern design. We are, however, not aware of such a clear demonstration of the reusability for other nanostructured SERS pattern, such as the commercial Klarite pattern. ${ }^{[10]}$

\section{Conclusions}

In summary, using EUV interference lithography, we prepared Au-templated nanoslit patterned substrates over $\mathrm{mm}^{2}$ large area in a two-step process, which were used for SERS measurements on molecular adsorbate assemblies. The substrates exhibited high SERS enhancement $\left(\sim 2^{*} 10^{6}\right)$ enabling the acquisition of high-quality Raman spectra of monomolecular films. The average enhancement factor possesses a high homogeneity (less than $3 \%$ deviation). Finally, no noticeable changes in the enhancement factor after several successive cleaning/self-assembly cycles were observed. All these features make the designed nanoslit-based substrate an ideal platform for an analytical tool or for advanced SERS experiments. In particular, because of the reusability and persisting characteristics, the electromagnetic enhancement can be kept constant while the chemical enhancement, ${ }^{[35]}$ the influence of molecular alignment, ${ }^{[36]}$ and surface selection rules ${ }^{[8]}$ can be studied experimentally upon the adsorption of customdesigned molecules.

\section{Acknowledgements}

This work has been financially supported by the Swiss Federal foundation. Part of this work was performed at the Swiss Light Source. We acknowledge the support of Y. Ekinci (PSI) and H. Solak (Eulitha AG). N.B. thanks T. A. Jung (PSI) for the support and helpful discussions. M. K. thanks A. B. Woodland (Frankfurt) for his help with recording a Raman spectrum of BPO. 


\section{Supporting information}

Supporting information may be found in the online version of this article.

\section{References}

[1] K. Kneipp, Y. Wang, H. Kneipp, L. T. Perelman, I. Itzkan, R. R. Dasari, M. S. Feld, Phys. Rev. Lett. 1997, 78, 1667.

[2] M. Rycenga, C. M. Cobley, J. Zeng, W. Li, C. H. Moran, Q. Zhang, D. Qin, Y. Xia, Chem. Rev. 2011, 111, 3669.

[3] K. A. Willets, R. P. Van Duyne, in Annual Review of Physical Chemistry, Vol. 58, 2007, pp. 267.

[4] Y. Zhu, R. A. Dluhy, Y. Zhao, Sensor Actuat B-Chem. 2011, 157, 42.

[5] H. Wang, C. S. Levin, N. J. Halas, J. Am. Chem. Soc. 2005, 127, 14992.

[6] H. Ko, S. Singamaneni, V. V. Tsukruk, Small 2008, 4, 1576.

[7] M. Moskovits, J. Raman Spectrosc. 2005, 36, 485.

[8] E. C. Le Ru, S. A. Meyer, C. Artur, P. G. Etchegoin, J. Grand, P. Lang, F. Maurel, Chem. Commun. 2011, 47, 3903-3905.

[9] M. J. Natan, Faraday Discuss. 2006, 132, 321.

[10] S. Sadate, Opt. Eng. 2010, 49, 106501.

[11] X. Li, H. Hu, D. Li, Z. Shen, Q. Xiong, S. Li, H. J. Fan, ACS Appl. Mater. Interfaces 2012, 4, 2180.

[12] S. M. Mahurin, J. John, M. J. Sepaniak, S. Dai, Appl. Spectrosc. 2011, 65, 417.

[13] G. Sinha, L. E. Depero, I. Alessandri, ACS Appl. Mater. Interfaces 2011, 3, 2557.

[14] U. S. Dinish, F. C. Yaw, A. Agarwal, M. Olivo, Biosens. Bioelectron. 2011, 26, 1987.

[15] D. Cialla, A. März, R. Böhme, F. Theil, K. Weber, M. Schmitt, J. Popp, Anal. Bioanal. Chem. 2011, 403, 1.

[16] H. Duan, H. Hu, K. Kumar, Z. Shen, J. K. W. Yang, ACS Nano 2011, 5, 7593.

[17] B. Päivänranta, A. Langner, E. Kirk, C. David, Y. Ekinci, Nanotechnology 2011, 22, 375302.

[18] H. J. Himmel, A. Terfort, C. Wöll, J. Am. Chem. Soc. 1998, 120, 12069.

[19] B. Schüpbach, M. Bolte, M. Zharnikov, A. Terfort, Eur. J. Org. Chem. 2010, 16, 3041.

[20] V. Auzelyte, C. Dais, P. Farquet, D. Grutzmacher, L. J. Heyderman, F. Luo, S. Olliges, C. Padeste, P. K. Sahoo, T. Thomson, A. Turchanin, C. David, H. H. Solak, J. Micro-Nanolith Mem. 2009, 8, 021204.
[21] T. Siegfried, Y. Ekinci, H. H. Solak, O. J. F. Martin, H. Sigg, Appl. Phys. Lett. 2011, 99, 263302.

[22] M. J. Frisch, G. W. Trucks, H. B. Schlegel, G. E. Scuseria, M. A. Robb, J. R. Cheeseman, G. Scalmani, V. Barone, B. Mennucci, G. A. Petersson, H. Nakatsuji, M. Caricato, X. Li, H. P. Hratchian, A. F. Izmaylov, J. Bloino, G. Zheng, J. L. Sonnenberg, M. Hada, M. Ehara, K. Toyota, R. Fukuda, J. Hasegawa, M. Ishida, T. Nakajima, Y. Honda, O. Kitao, H. Nakai, T. Vreven, J. A. Montgomery, J. E. Peralta, F. Ogliaro, M. Bearpark, J. J. Heyd, E. Brothers, K. N. Kudin, V. N. Staroverov, R. Kobayashi, J. Normand, K. Raghavachari, A. Rendell, J. C. Burant, S. S. lyengar, J. Tomasi, M. Cossi, N. Rega, J. M. Millam, M. Klene, J. E. Knox, J. B. Cross, V. Bakken, C. Adamo, J. Jaramillo, R. Gomperts, R. E. Stratmann, O. Yazyev, A. J. Austin, R. Cammi, C. Pomelli, J. W. Ochterski, R. L. Martin, K. Morokuma, V. G. Zakrzewski, G. A. Voth, P. Salvador, J. J. Dannenberg, S. Dapprich, A. D. Daniels, Ö. Farkas, J. B. Foresman, J. V. Ortiz, J. Cioslowski, D. J. Fox, in Gaussian 09, Revision A.02, Gaussian, Inc., Wallingford CT, Wallingford $C T, 2009$.

[23] Z. Weihua, H. Fischer, T. Schmid, R. Zenobi, O. J. F. Martin, J. Phys. Chem. C 2009, 113, 14672.

[24] M. Kind, C. Woll, Prog. Surf. Sci. 2009, 84, 230.

[25] J. C. Love, L. A. Estroff, J. K. Kriebel, R. G. Nuzzo, G. M. Whitesides, Chem. Rev. 2005, 105, 1103.

[26] F. Chesneau, B. Schupbach, K. Szelagowska-Kunstman, N. Ballav, P. Cyganik, A. Terfort, M. Zharnikov, Phys. Chem. Chem. Phys. 2010, $12,12123$.

[27] A. Shaporenko, M. Brunnbauer, A. Terfort, M. Grunze, M. Zharnikov, J. Phys. Chem. B 2004, 108, 14462.

[28] T. Winkler, N. Ballav, H. Thomas, M. Zharnikov, A. Terfort, Angew. Chem. Int. Ed. 2008, 47, 7238.

[29] H. G. M. Edwards, A. F. Johnson, I. R. Lewis, D. J. Maitland, N. Webb, J. Mol. Struct. 1992, 268, 363.

[30] E. C. Le Ru, M. Meyer, E. Blackie, P. G. Etchegoin, J. Raman Spectrosc. 2008, 39, 1127.

[31] M. Moskovits, J. Chem. Phys. 1982, 77, 4408.

[32] A. T. Zayak, Y. S. Hu, H. Choo, J. Bokor, S. Cabrini, P. J. Schuck, J. B. Neaton, Phys. Rev. Lett. 2011, 106, 083003.

[33] D. Bhandari, S. M. Wells, A. Polemi, I. I. Kravchenko, K. L. Shuford, M. J. Sepaniak, J. Raman Spectrosc. 2008, 42, 1916.

[34] R. L. Aggarwal, L. W. Farrar, E. D. Diebold, D. L. Polla, J. Raman Spectrosc. 2009, 40, 1331.

[35] A. Barhoumi, D. Zhang, N. J. Halas, J. Am. Chem. Soc. 2008, 130, 14040.

[36] S. M. Morton, L. Jensen, J. Am. Chem. Soc. 2009, 131, 4090. 